\title{
Determinantes del empleo no agrícola y de los ingresos no agrícolas en el Ecuador
}

\author{
Cristian Vasco y Grace Natalie Tamayo
}

\section{Resumen}

Este artículo analiza los determinantes de la participación en el empleo no agrícola y de los ingresos no agrícolas en el Ecuador. Los resultados obtenidos con el método de estimación en dos etapas de Dubin y McFadden muestran que las mujeres son más propensas que los hombres a dedicarse al autoempleo no agrícola pero ganan mucho menos que los hombres empleados en el sector no agrícola. El empleo asalariado no agrícola es una opción común entre las personas más instruidas provenientes de hogares sin tierras, mientras que el empleo asalariado agrícola parece ser la única fuente de empleo para las personas que carecen de tierras e instrucción. La participación en el trabajo no agrícola es más probable en áreas situadas cerca de ciudades medianas con economías dinámicas. Finalmente, se observan diferencias regionales en los patrones de empleo que parecen estar relacionadas con la disponibilidad y la calidad de la tierra.

\section{Palabras clave}

Empleo rural, ingresos, zonas rurales, análisis matemático, modelos econométricos, Ecuador Clasificación JEL

P25, R11, R58

Autores

Cristian Vasco es Profesor Titular de la Facultad de Ciencias Agrícolas de la Universidad Central del Ecuador. clvasco@uce.edu.ec

Grace Natalie Tamayo es Coordinadora Académica de la Facultad de Ciencias Administrativas y Económicas de la Universidad Tecnológica Indoamérica, Ecuador. grace.tamayo@ indoamerica.edu.ec 


\section{Introducción}

El empleo rural no agrícola (ERNA) es importante para la subsistencia de la población rural en los países en desarrollo. En América Latina, este genera más del 40\% de los ingresos de los hogares rurales y emplea alrededor del 35\% de la población rural adulta (Köbrich y Dirven, 2007).

El ERNA se vincula ampliamente con la reducción de la pobreza (Elbers y Lanjouw, 2001; Lanjouw y Lanjouw, 2001), la reducción de los riesgos (Ellis, 2000; Haggblade, Hazell y Reardon, 2010), el mejoramiento de los ingresos (Haggblade, Hazell y Reardon, 2010) y una menor presión sobre los recursos naturales (Ellis, 1993). En consecuencia, comprender la naturaleza y los patrones de la participación en el ERNA constituye un primer paso para evaluar su potencial como herramienta de desarrollo rural.

Los factores que impulsan la participación en el ERNA en América Latina han sido objeto de una serie de estudios empíricos. En Chile, Berdegué y otros (2001) hallaron que los hogares encabezados por mujeres con un buen nivel educativo y acceso a crédito eran más propensos a participar en el trabajo no agrícola. Laszlo (2005) llegó a la misma conclusión sobre los hogares peruanos situados en los distritos con más centros poblados y un sector turístico más desarrollado. En Nicaragua, Isgut (2004) determinó que el trabajo asalariado fuera del predio agrícola era realizado principalmente por hombres con un bajo nivel educativo. En contraste, el autoempleo no agrícola era común entre las mujeres, mientras que el trabajo asalariado no agrícola era realizado sobre todo por las personas más instruidas. Ferreira y Lanjouw (2001) estudiaron los determinantes del trabajo no agrícola en el nordeste del Brasil y hallaron que los hombres instruidos eran más propensos a dedicarse a trabajos no agrícolas bien remunerados, mientras que las mujeres se dedicaban a empleos no agrícolas de baja remuneración. También en el Brasil, Jonasson y Helfand (2010) hallaron que la probabilidad de participación en el ERNA era más alta cerca de los centros poblados.

Son pocos los estudios en los que se examinan los determinantes de la participación y los ingresos en el empleo no agrícola en el Ecuador. Sobre la base de datos del Estudio sobre la Medición de Niveles de Vida de 1995, Elbers y Lanjouw (2001) hallaron que el empleo rural no agrícola correspondía sobre todo a mujeres instruidas provenientes de hogares que no se dedicaban a cultivar la tierra. Sin embargo, la situación cambiaba en el caso de los empleos no agrícolas de alta productividad, que estaban principalmente en manos de los hombres. Lanjouw (1999) encontró que los hogares instruidos que no se dedicaban a la agricultura y tenían acceso a electricidad y una línea telefónica eran más propensos a poseer empresas rurales. Sin embargo, la medida en que el ERNA ha cambiado y evolucionado desde la década de 1990 es una pregunta que aún se debe responder.

A partir de los datos de la Encuesta Nacional de Empleo, Desempleo y Subempleo de 2010, en este trabajo se analizan los determinantes del empleo no agrícola y de los ingresos no agrícolas en el Ecuador. A diferencia de investigaciones previas en el Ecuador y América Latina, se basa en el método de estimación en dos etapas de Dubin y McFadden, que controla tanto la simultaneidad en las decisiones con respecto a la principal fuente de ingresos de una persona como el posible sesgo de selección en las regresiones de ingresos. Los resultados indican que el empleo no agrícola constituye la principal fuente de ingresos para una fracción importante (36\%) de la población rural del Ecuador. Las personas instruidas de los hogares más ricos prefieren dedicarse al empleo asalariado no agrícola. Por el contrario, el empleo asalariado agrícola es la (única) opción para las personas sin instrucción de los hogares más pobres. La falta de tierras parece ser uno de los factores que impulsan a la población rural hacia el empleo no agrícola. Si bien las mujeres son más propensas a obtener sus ingresos del autoempleo no agrícola, estos son más bajos que los de los hombres. El empleo no agrícola florece en las áreas cercanas a las ciudades medianas que cuentan con un sector agrícola dinámico. 
El trabajo se divide en seis secciones, incluida esta Introducción. En la segunda se presenta el marco teórico y en la tercera se detallan los datos y las variables utilizadas. En la cuarta sección se describe la estrategia empírica, mientras que en la quinta se presentan y analizan los resultados. En la sexta y última sección se formulan las conclusiones.

\section{Marco teórico}

El modelo de subsistencia rural (Ellis, 1999) es un buen punto de partida para analizar las decisiones relativas a la diversificación de los ingresos. Los hogares rurales tratan de maximizar sus rendimientos sujetos a algunas limitaciones, entre ellas el dinero en efectivo, el tiempo y la tecnología. De acuerdo con este modelo, la diversificación es una función de los rendimientos del tiempo de trabajo dedicado a las actividades dentro del predio agrícola en comparación con el empleo fuera del predio. Con una cantidad fija de activos agrícolas (tierra e infraestructura) y tiempo de trabajo, los hogares comparan los rendimientos y eligen entre destinar más tiempo al trabajo agrícola o invertirlo en actividades no agrícolas. En la práctica, el modelo de subsistencia rural sostiene que los hogares toman las decisiones con respecto a la diversificación de los medios de subsistencia según las diferentes dotaciones de capital natural (tierra, agua, árboles), capital físico (canales de irrigación, implementos, carreteras), capital humano (educación, aptitudes, salud), capital financiero o sus sustitutos (dinero en efectivo, ahorros, ganado) y capital social (redes, asociaciones).

Las decisiones de diversificación están determinadas por factores de atracción y expulsión. Los hogares o las personas se dedican al ERNA a condición de que produzca rendimientos más altos que las actividades agrícolas, controlando el riesgo (Reardon y otros, 2000). Esos rendimientos constituyen el factor de atracción. Esto ocurre sobre todo en las regiones donde las actividades agrícolas, mineras y turísticas exitosas confieren dinamismo a las economías locales. Por otra parte, los factores de expulsión son los riesgos relativos a los ingresos derivados de varios factores, incluidos los riesgos climáticos, la escasez de tierras y las fallas del mercado. En la literatura se distingue entre las estrategias para gestionar los riesgos (ex ante) y las estrategias para hacerles frente (ex post) (Reardon y otros, 2000). En el primer caso, los hogares optan voluntariamente por diversificar las fuentes de ingresos para prevenir posibles problemas, mientras que en el segundo los hogares diversifican para hacer frente a acontecimientos inesperados que amenazan su subsistencia. Asimismo, Reardon y otros (2000) enumeran un conjunto de variables de capacidad - entre ellas el capital humano, financiero, social y físico - que los hogares o las personas necesitan para dedicarse a las actividades no agrícolas.

\section{Datos y variables}

La principal fuente de datos es la Encuesta Nacional de Empleo, Desempleo y Subempleo de 2010, realizada por el Instituto Nacional de Estadística y Censos (INEC). La encuesta es representativa a nivel nacional e incluye información acerca del empleo, los ingresos y la vivienda de 82.774 habitantes de zonas urbanas y rurales. El presente estudio se concentra en una submuestra de 16.014 personas residentes en áreas rurales, de 15 años de edad o más, que tenían un trabajo (incluso no remunerado) en el momento de la encuesta. En el contexto de este estudio, el ERNA se define como cualquier ocupación distinta del autoempleo agrícola y el empleo asalariado agrícola.

Las definiciones y estadísticas descriptivas de las variables utilizadas en el análisis empírico se presentan en el cuadro 1. Las variables dependientes son cuatro variables dicotómicas que indican si la ocupación principal de una persona corresponde a: autoempleo agrícola, empleo asalariado 
agrícola, autoempleo no agrícola o empleo asalariado no agrícola. Uno de los objetivos de este estudio es analizar los determinantes de los ingresos de las actividades no agrícolas. Los predictores incluyen un conjunto de características individuales, del hogar, parroquiales y regionales que se describen a continuación. Las variables individuales incluyen edad, género, jefatura del hogar, educación y etnia. Se espera que los rendimientos de la educación sean mayores en el sector no agrícola. Para controlar este efecto se incluyen en la especificación tres variables dicotómicas que toman el valor 1 si la persona ha completado la educación primaria, secundaria o universitaria, respectivamente. En el Ecuador rural, el género puede estar relacionado no solo con la discriminación en materia de ingresos sino también con la discriminación en la división del trabajo (Martínez, 2000). Por ese motivo, se incluyó en el modelo una variable ficticia que toma el valor 1 para las mujeres. La etnia se controla mediante cuatro variables ficticias que indican si una persona se identifica como indígena, afroecuatoriana, montubia ${ }^{1}$ o blanca. El grupo mestizo, el más grande de la muestra, se utiliza como grupo excluido. El modelo también incluye una variable ficticia que indica si la persona es el jefe o la jefa del hogar.

Las características del hogar incluyen variables demográficas, riqueza y propiedad de tierras. Los indicadores demográficos de los hogares comprenden una variable ficticia que toma el valor 1 si el jefe del hogar es una mujer y el número de niños, niñas, hombres adultos y mujeres adultas en el hogar (véanse las definiciones en el cuadro 1). Se espera que estas variables influyan en las decisiones de empleo individuales. Para controlar la riqueza se incluye en la especificación un índice construido a partir de determinados activos del hogar². La riqueza no solo puede conferir la capacidad de superar barreras de ingreso a la participación en actividades no agrícolas de alto rendimiento, sino que también puede asociarse con mayores niveles de capital social, que puede ser útil en la búsqueda de un empleo no agrícola. El tamaño de las tierras poseídas es otro determinante significativo de la participación en el trabajo no agrícola (Elbers y Lanjouw, 2001), pero lamentablemente la Encuesta Nacional de Empleo, Desempleo y Subempleo de 2010 no incluye información al respecto. Como alternativa se agregó al modelo una variable ficticia que toma el valor 1 si el hogar posee tierras.

En general, la disponibilidad de electricidad y teléfono presenta una correlación positiva con el ERNA (Lanjouw, 1999; Elbers y Lanjouw, 2001). Para tener esto en cuenta, se incluye en la lista de predictores la proporción de hogares rurales con acceso a conexión eléctrica y línea telefónica (estimada a nivel parroquial sobre la base del Censo de Población y Vivienda 2010). La distancia con respecto a los mercados circundantes y su tamaño también resultaron importantes determinantes del empleo no agrícola en el pasado (Jonasson y Helfand, 2010). Para probar esta hipótesis en el caso ecuatoriano se incluyeron en el modelo las distancias por carretera ${ }^{3}$ de una parroquia con respecto a la ciudad más cercana con una población de 50.000-100.000, 100.000-250.000, 250.000-500.000 y más de 500.000 habitantes. Es de esperar que a medida que aumenta la distancia disminuya la probabilidad de que una persona trabaje en el sector no agrícola. Por último, las diferencias regionales se controlan mediante dos variables ficticias que toman el valor 1 si la persona vive en la región de la Costa o en la región Oriente, respectivamente. Las personas que residen en la Sierra, el grupo más grande de la muestra, constituyen el grupo de referencia.

1 Los montubios son un grupo étnico formado por campesinos que viven exclusivamente en el litoral ecuatoriano.

2 Este índice es el primer componente principal de las siguientes variables: propiedad de vivienda y automóvil, disponibilidad de agua corriente y ducha interna y número de televisores, reproductores de DVD, radios, computadoras, teléfonos móviles, refrigeradores y cocinas. El primer componente principal representa alrededor del $32 \%$ de la variación.

3 Las distancias se basan en datos del Ministerio de Transporte y Obras Públicas y los gobiernos provinciales. En el caso de dos islas y tres parroquias sin conexión por carretera se tomaron las distancias en línea recta hasta la parroquia más cercana con una conexión por carretera y se sumaron a la distancia por carretera desde esa parroquia hasta la ciudad más cercana con una población de 50.000-100.000, 100.000-250.000, 250.000-500.000 y más de 500.000 habitantes, respectivamente. 
Cuadro 1

Variables y estadísticas descriptivas

\begin{tabular}{|c|c|c|c|}
\hline Variable & Descripción & Media & $\begin{array}{l}\text { Desviación } \\
\text { estándar }\end{array}$ \\
\hline \multicolumn{4}{|l|}{ Variables dependientes } \\
\hline FSE & Autoempleo agrícola como ocupación principal (0/1) & 0,453 & 0,497 \\
\hline FWE & Empleo asalariado agrícola como ocupación principal (0/1) & 0,211 & 0,408 \\
\hline NFSE & Autoempleo no agrícola como ocupación principal (0/1) & 0,104 & 0,305 \\
\hline NFWE & Empleo asalariado no agrícola como ocupación principal (0/1) & 0,230 & 0,421 \\
\hline Ingresos no agrícolas & Log de ingresos no agrícolas (en dólares) & 5,297 & 0,854 \\
\hline \multicolumn{4}{|l|}{ Variables individuales } \\
\hline Edad & Edad de la persona (en años) & 41,554 & 17,426 \\
\hline Mujer & La persona es una mujer (0/1) & 0,334 & 0,441 \\
\hline Jefatura del hogar & La persona es jefe del hogar (0/1) & 0,466 & 0,498 \\
\hline Educación primaria & La persona completó la enseñanza primaria (0/1) & 0,516 & 0,499 \\
\hline Educación secundaria & La persona completó la enseñanza secundaria (0/1) & 0,024 & 0,154 \\
\hline Educación universitaria & La persona completó la universidad (0/1) & 0,024 & 0,153 \\
\hline Indígena & La persona es indígena $(0 / 1)$ & 0,157 & 0,364 \\
\hline Afroecuatoriano & La persona es afroecuatoriana (0/1) & 0,017 & 0,131 \\
\hline Montubio & La persona es montubia (0/1) & 0,084 & 0,278 \\
\hline Blanco & La persona es blanca $(0 / 1)$ & 0,018 & 0,134 \\
\hline \multicolumn{4}{|l|}{ Variables del hogar } \\
\hline Jefatura femenina & El hogar está encabezado por una mujer (0/1) & 0,162 & 0,368 \\
\hline Niños & Número de varones menores de 15 años & 0,847 & 1,075 \\
\hline Niñas & Número de mujeres menores de 15 años & 0,796 & 1,065 \\
\hline Hombres adultos & Número de varones de 15 años o más & 1,806 & 1,125 \\
\hline Mujeres adultas & Número de mujeres de 15 años o más & 1,654 & 0,979 \\
\hline Riqueza & Índice de riqueza & 0,244 & 2,065 \\
\hline Propiedad de tierras & El hogar posee tierras $(0 / 1)$ & 0,481 & 0,499 \\
\hline \multicolumn{4}{|c|}{ Variables parroquiales y regionales } \\
\hline Electricidad & Proporción de hogares con acceso a electricidad (censo de 2010) & 0,885 & 0,136 \\
\hline Teléfono & Proporción de hogares con acceso a teléfono (censo de 2010) & 0,179 & 0,151 \\
\hline Distancia 1 & $\begin{array}{l}\text { Log de la distancia hasta la ciudad más cercana con } \\
50.000-100.000 \text { habitantes (en kilómetros) }\end{array}$ & 3,065 & 1,714 \\
\hline Distancia 2 & $\begin{array}{l}\text { Log de la distancia hasta la ciudad más cercana con } \\
\text { 100.000-250.000 habitantes (en kilómetros) }\end{array}$ & 3,649 & 1,688 \\
\hline Distancia 3 & $\begin{array}{l}\text { Log de la distancia hasta la ciudad más cercana con } \\
250.000-500.000 \text { habitantes (en kilómetros) }\end{array}$ & 4,273 & 1,718 \\
\hline Distancia 4 & $\begin{array}{l}\text { Log de la distancia hasta la ciudad más cercana con } \\
\text { más de } 500.000 \text { habitantes (en kilómetros) }\end{array}$ & 4,917 & 1,055 \\
\hline Costa & La persona reside en la región de la Costa (0/1) & 0,358 & 0,479 \\
\hline Oriente & La persona reside en la región Oriente (0/1) & 0,052 & 0,223 \\
\hline
\end{tabular}

Fuente: Elaboración propia, sobre la base de datos de la Encuesta Nacional de Empleo, Desempleo y Subempleo, diciembre de 2010 y Censo de Población y Vivienda 2010.

Nota: (1/0) identifica las variables ficticias. 


\section{Estrategia empírica}

La elección de la categoría de trabajo y los ingresos de la ocupación principal se obtienen mediante el método de estimación en dos etapas de Dubin y McFadden (Dubin y McFadden, 1984). En la primera etapa, la probabilidad de que una persona se encuentre en la categoría de autoempleo agrícola, empleo asalariado agrícola, autoempleo no agrícola o empleo asalariado no agrícola se estima utilizando un modelo multinomial logit. En la segunda etapa se estiman los determinantes de los ingresos individuales durante el mes de noviembre de 2010, en virtud de la categoría de empleo elegida en la primera etapa. Para controlar una posible correlación de errores entre las dos etapas, la segunda etapa incluye un término de corrección de selección calculado a partir de la primera.

En este contexto, las personas eligen una categoría de empleo $c$ entre una serie de alternativas $M$ basadas en la utilidad condicional latente $E_{c}{ }^{*}$.

$$
E_{c}^{*}=z_{c} \gamma_{c}+\eta_{c}, \quad c=1, \ldots, M
$$

donde $z_{c}$ representa un vector de las variables individuales, del hogar y parroquiales descritas; $\gamma_{c}$ es un vector de estimadores y $\eta_{c}$ es el término de perturbación. $E_{c}$ toma el valor 1 si se elige la categoría de empleo $c$ y 0 en caso contrario.

Para la categoría elegida, el log de los ingresos $(y)$ se modela de la siguiente manera:

$$
y_{1}=x_{1} \beta_{1}+u_{1}
$$

donde $x$ es un vector de variables explicativas que afectan a $y$, y $u$ representa un término de perturbación con las siguientes propiedades: $E\left(u_{1} \mid x, z\right)=0$ y $V\left(u_{1} \mid x, z\right)=\sigma^{2}$. Se asume que el modelo se identifica excluyendo algunas de las variables en $z$ de las variables en $x$. La variable dependiente $y_{1}$ se observa solo cuando se selecciona la categoría de empleo 1. Esto ocurre cuando:

$$
\begin{gathered}
E_{1}^{*}>\max _{c \neq 1}\left(E_{c}^{*}\right) \\
\varepsilon_{1}=\max _{c \neq 1}\left(E_{c}^{*}-E_{1}\right) ; \varepsilon_{1}<0
\end{gathered}
$$

Al asumir que $\eta_{c}$ es independiente y está idénticamente distribuido con distribución de Gumbel, las funciones acumulativas y de densidad son: $G(\eta)=\exp \left(-e^{-\eta}\right)$ y $g(\eta)=\exp \left(-\eta-e^{-\eta}\right)$, respectivamente. De acuerdo con McFadden (1973), esta especificación permite estimar la probabilidad de elección de la categoría de empleo 1 utilizando un modelo multinomial logit con la siguiente forma:

$$
P\left(z_{1} \gamma_{1}>\varepsilon_{1}\right)=\frac{\exp \left(z_{1} \gamma_{1}\right)}{\sum_{c=1} \exp \left(z_{c} \gamma_{c}\right)}
$$

En la segunda parte del análisis se examinan los ingresos en cada categoría de empleo, haciendo hincapié en el autoempleo no agrícola y en el empleo asalariado no agrícola. Es posible que las personas de una categoría difieran considerablemente de las de las otras categorías y que esas diferencias incidan en los ingresos. Este defecto se señala en varios estudios (Elbers y Lanjouw, 2001; Lanjouw, 2001; Isgut, 2004; Jonasson y Helfand, 2010; Atamanova y Van den Berg, 2012), en los que se utilizan métodos de corrección del sesgo de selección al modelar los ingresos del empleo 
no agrícola. Al igual que el método en dos etapas de Heckman, el enfoque de Dubin y McFadden también controla la selectividad de la muestra. Sin embargo, la existencia de varias opciones supone varios términos de corrección, en este caso uno por cada categoría de empleo. Para estimar los determinantes de los ingresos en cada categoría, en este estudio se aplica la variante del método de corrección de Dubin y McFadden propuesta por Bourguignon, Fournier y Gurgand (2007):

$$
y_{1}=x_{1} \beta_{1}+\sigma\left[\rho_{1} m\left(P_{1}\right)+\sum_{c=2 \ldots M} \rho_{c} \frac{P_{c}}{P_{c}-1} m\left(P_{c}\right)\right]+v_{c}
$$

donde $m\left(P_{c}\right)$ y $m\left(P_{1}\right)$ son las probabilidades, $\left(\sigma \rho_{1}\right), \ldots,\left(\sigma \rho_{c}\right)$ son los términos del coeficiente para la corrección policotómica del sesgo de selección y $v_{c}$ es un parámetro de error ortogonal que tiene una expectativa media cero. Este enfoque tiene la ventaja de funcionar bien incluso si se infringe el supuesto de independencia de alternativas irrelevantes (Bourguignon, Fournier y Gurgand, 2007).

\section{Resultados y análisis}

\section{Análisis descriptivo}

En el cuadro 2 se muestra la proporción de la población rural dedicada al ERNA por región y sector. Mientras que el comercio es más importante en la Costa (litoral), la industria manufacturera y la construcción emplean a un porcentaje significativo de la población activa en la Sierra (región andina). El porcentaje de la población que se dedica a la enseñanza y al servicio público es mayor en el Oriente (región amazónica) que en la Costa o la Sierra. Algo similar ocurre con el sector minero, pues los campos petroleros están situados en el Oriente. En promedio, el 33,5\% de la mano de obra rural ecuatoriana tiene un empleo no agrícola. Los patrones de distribución de la mano de obra entre el autoempleo agrícola, el empleo asalariado agrícola, el autoempleo no agrícola y el empleo asalariado no agrícola por región geográfica se presentan en el gráfico 1. El porcentaje de personas cuya ocupación principal es el empleo asalariado agrícola es mucho mayor en la Costa que en la Sierra y en el Oriente. La proporción de personas en el ERNA no parece variar mucho entre las regiones. Estos resultados son coherentes con las proporciones de ingresos obtenidos por categoría de empleo (véase el gráfico 2). Mientras que el autoempleo agrícola representa más del $40 \%$ de los ingresos de los hogares en la Sierra y el Oriente, este porcentaje desciende al 32\% en la Costa. En contraste, la proporción de ingresos de los hogares correspondiente al empleo asalariado agrícola es más del doble en la Costa que en la Sierra o el Oriente.

Cuadro 2

Ecuador: empleo rural no agrícola por región y sector

(En porcentajes)

\begin{tabular}{lccc}
\hline & Costa & Sierra & Oriente \\
\hline Comercio & 13,7 & 8,5 & 9,0 \\
\hline Transporte y comunicaciones & 2,1 & 2,8 & 1,5 \\
\hline Finanzas & 0,1 & 0,1 & 0,0 \\
\hline Propiedades & 0,9 & 0,7 & 0,7 \\
\hline Servicio público & 1,0 & 1,4 & 4,1 \\
\hline Enseñanza & 1,9 & 2,0 & 5,9 \\
\hline Servicios de salud & 0,8 & 0,7 & 2,0 \\
\hline
\end{tabular}


Cuadro 2 (conclusión)

\begin{tabular}{lccc}
\hline & Costa & Sierra & Oriente \\
\hline Minería & 1,6 & 0,3 & 3,1 \\
\hline Industria manufacturera & 3,5 & 8,7 & 2,6 \\
\hline Construcción & 3,6 & 5,7 & 4,3 \\
\hline Servicio doméstico & 1,4 & 2,1 & 1,1 \\
\hline Otros & 1,2 & 1,3 & 1,7 \\
\hline
\end{tabular}

Fuente: Elaboración propia, sobre la base de datos de la Encuesta Nacional de Empleo, Desempleo y Subempleo, diciembre de 2010.

Gráfico 1

Ecuador: ocupación principal por categoría de empleo y región

(En porcentajes)

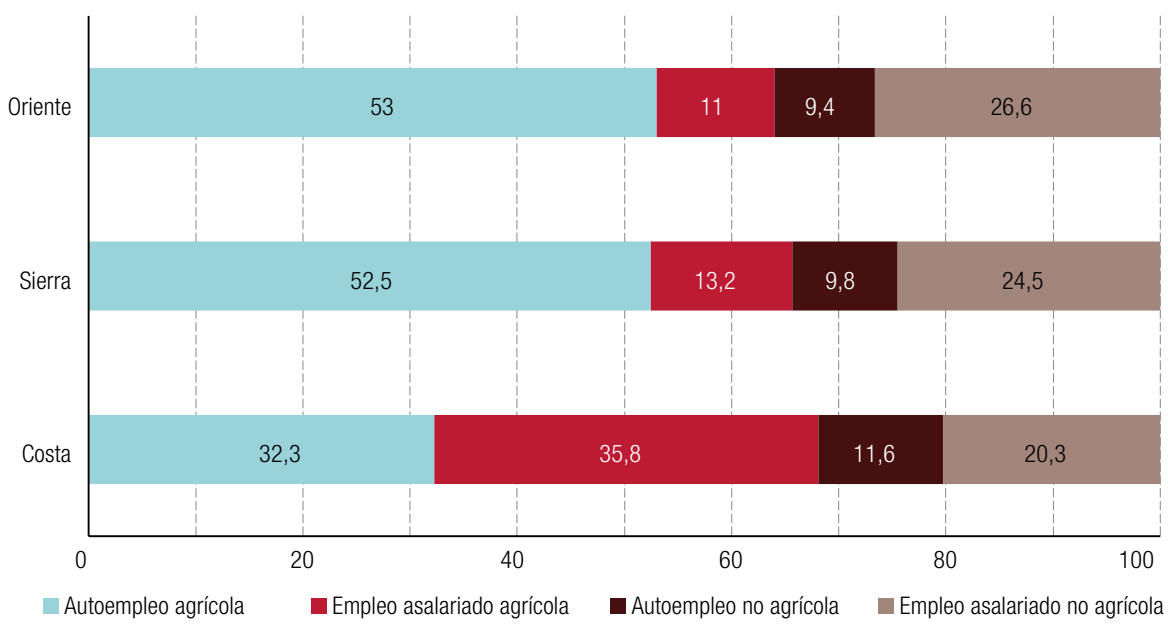

Fuente: Elaboración propia, sobre la base de datos de la Encuesta Nacional de Empleo, Desempleo y Subempleo, diciembre de 2010.

\section{Gráfico 2}

Ecuador: ingresos de los hogares por categoría de empleo y región (En porcentajes)

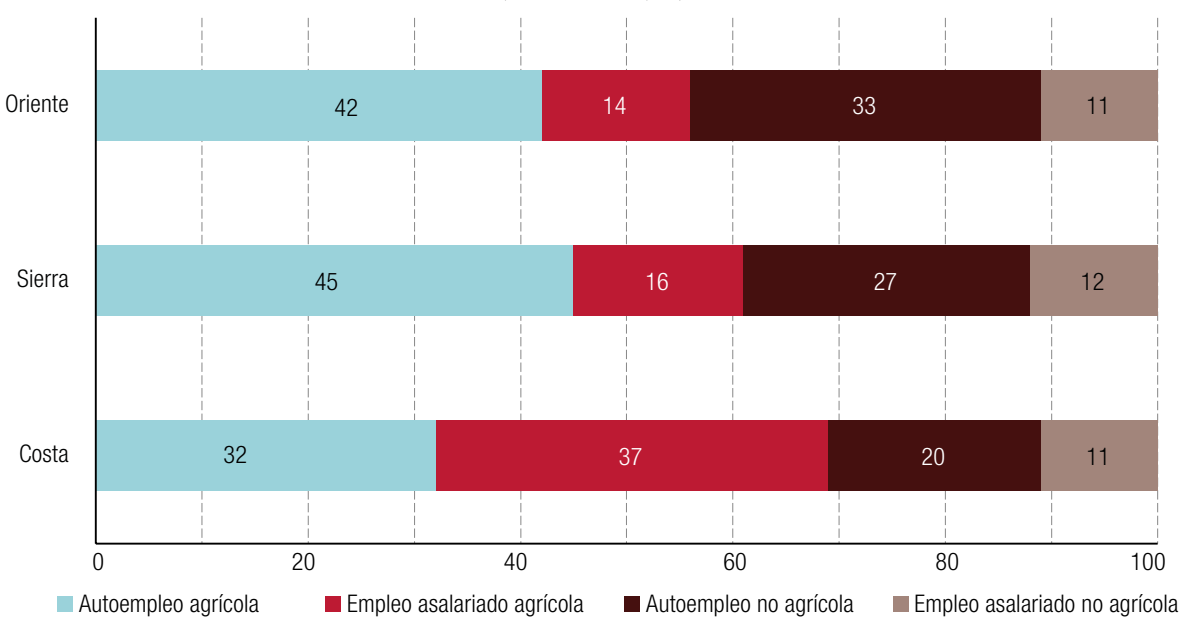

Fuente: Elaboración propia, sobre la base de datos de la Encuesta Nacional de Empleo, Desempleo y Subempleo, diciembre de 2010. 
En el cuadro 3 se presentan los ingresos medios por región y subsector. Los salarios varían mucho más entre los sectores que entre las regiones. Sin embargo, en el caso de la agricultura, los ingresos derivados del autoempleo agrícola y del empleo asalariado agrícola son más altos en la Costa, probablemente porque la mayoría de los productos agrícolas exportables se producen en esa región. En el otro extremo, los ingresos derivados de la agricultura son más bajos en el Oriente, especialmente en el autoempleo. Los ingresos no agrícolas son más altos que los ingresos agrícolas en todas partes excepto en la Costa. Las cifras más altas corresponden al servicio público, la enseñanza y, en particular, la minería. Los salarios del comercio y la enseñanza son más altos en el Oriente. Esto puede reflejar una escasez de trabajadores calificados en esos sectores, que se traduce en un incremento de los salarios en esa área. Los trabajadores de la industria minera ganan mucho más en el Oriente que en la Sierra y la Costa, un dato que no sorprende dado que, como ya se mencionó, la explotación petrolera tiene lugar en esa zona.

Cuadro 3

Ecuador: ingresos rurales individuales derivados de la ocupación primaria, media mensual por región y sector

(En dólares)

\begin{tabular}{|c|c|c|c|c|c|c|}
\hline & \multicolumn{2}{|c|}{ Costa } & \multicolumn{2}{|c|}{ Sierra } & \multicolumn{2}{|c|}{ Oriente } \\
\hline & Autoempleo & $\begin{array}{c}\text { Empleo } \\
\text { asalariado }\end{array}$ & Autoempleo & $\begin{array}{c}\text { Empleo } \\
\text { asalariado }\end{array}$ & Autoempleo & $\begin{array}{c}\text { Empleo } \\
\text { asalariado }\end{array}$ \\
\hline Agricultura & 283 & 185 & 160 & 151 & 139 & 172 \\
\hline Comercio & 192 & 226 & 279 & 245 & 254 & 259 \\
\hline Industria manufacturera & 152 & 261 & 198 & 246 & 184 & 213 \\
\hline Construcción & 263 & 257 & 282 & 267 & - & 268 \\
\hline Servicio público & - & 516 & - & 675 & - & 621 \\
\hline Enseñanza & - & 372 & - & 457 & - & 475 \\
\hline Minería & 290 & 339 & 264 & 342 & 196 & 630 \\
\hline
\end{tabular}

Fuente: Elaboración propia, sobre la base de datos de la Encuesta Nacional de Empleo, Desempleo y Subempleo, diciembre de 2010.

\section{Análisis econométrico}

En primer lugar, el supuesto de independencia de alternativas irrelevantes se examina mediante la prueba de Hausman (suest-based Hausman test). La prueba no rechaza la hipótesis nula de independencia de alternativas, de manera que no se infringe el supuesto de independencia de alternativas irrelevantes.

En el cuadro 4 se presentan los efectos marginales de un modelo multinomial logit con cuatro resultados posibles. En comparación con los hombres, las mujeres son más propensas a trabajar por cuenta propia (autoempleo agrícola o no agrícola) y menos propensas a dedicarse al empleo asalariado agrícola. El trabajo agrícola tiene lugar cerca del hogar, de modo que es más fácil para ellas combinar la crianza de los niños con el empleo por cuenta propia en ese sector. Además, en el contexto del Ecuador rural, se acepta culturalmente que las mujeres realicen trabajo agrícola (Martínez, 2000). En investigaciones previas (Lanjouw, 1999; Elbers y Lanjouw, 2001) se concluyó que las mujeres eran más propensas a participar en el ERNA. Sin embargo, cuando el ERNA se divide en autoempleo no agrícola y empleo asalariado no agrícola, la variable ficticia de género presenta un efecto significativo solo con respecto a la primera categoría. Estos resultados concuerdan con una serie de estudios cualitativos (Martínez, 2000, 2002 y 2004) que sugieren que los hombres se dedican al trabajo fuera del predio, ya sea agrícola o de otro tipo, mientras que las mujeres se dedican a la parcela familiar y a las actividades no agrícolas (comercio minorista y fabricación de artesanías). 
Cuadro 4

Ecuador: determinantes del tipo de ocupación principal en las áreas rurales (efectos marginales)

\begin{tabular}{|c|c|c|c|c|}
\hline Variable & $\begin{array}{l}\text { Autoempleo } \\
\text { agrícola }\end{array}$ & $\begin{array}{c}\text { Empleo asalariado } \\
\text { agrícola }\end{array}$ & $\begin{array}{c}\text { Autoempleo } \\
\text { no agrícola }\end{array}$ & $\begin{array}{c}\text { Empleo asalariado } \\
\text { no agrícola }\end{array}$ \\
\hline \multicolumn{5}{|l|}{ Variables individuales } \\
\hline Edad & $-0,007^{\star \star \star}$ & $-0,000$ & $0,006^{\star \star \star}$ & 0,001 \\
\hline Edad al cuadrado & $0,000^{\star \star \star}$ & 0,000 & $-0,000^{\star \star \star}$ & $-0,000^{\star \star \star}$ \\
\hline Mujer & $0,094^{\star \star \star}$ & $-0,165^{\star \star \star}$ & $0,086^{\star \star \star}$ & 0,015 \\
\hline Jefatura del hogar & 0,017 & $-0,025^{\star \star \star}$ & $0,012^{*}$ & $-0,004$ \\
\hline Educación primaria & $-0,017^{\star}$ & $-0,018^{\star \star \star}$ & $0,011^{\star *}$ & 0,010 \\
\hline Educación secundaria & $-0,031$ & $-0,127^{\star \star \star}$ & 0,011 & $0,147^{\star \star \star}$ \\
\hline Educación universitaria & $-0,329^{\star \star \star}$ & $-0,164^{\star \star \star}$ & $-0,026^{\star \star}$ & $0,520^{\star \star *}$ \\
\hline Indígena & $0,089^{\star \star \star}$ & $-0,119^{\star \star \star}$ & $-0,015$ & 0,046 \\
\hline Afroecuatoriano & $-0,005$ & $-0,012$ & $-0,021$ & 0,040 \\
\hline Montubio & $0,069^{\star \star \star}$ & $0,028^{\star \star \star}$ & $-0,032^{\star \star \star}$ & $-0,064^{\star \star \star}$ \\
\hline Blanco & $-0,030$ & $-0,024$ & 0,029 & 0,025 \\
\hline \multicolumn{5}{|l|}{ Variables del hogar } \\
\hline Jefatura femenina & $-0,070^{\star * *}$ & 0,008 & $0,019^{\star \star}$ & $0,042^{* \star \star}$ \\
\hline Niños & 0,002 & 0,001 & 0,016 & $-0,005$ \\
\hline Niñas & 0,002 & 0,003 & 0,016 & $-0,007$ \\
\hline Hombres adultos & $-0,002$ & $0,017^{\star \star \star}$ & $-0,010^{\star \star \star}$ & $-0,009^{\star \star}$ \\
\hline Mujeres adultas & $-0,013^{\star \star \star}$ & 0,000 & $-0,007^{\star \star \star}$ & $0,020^{\star * \star}$ \\
\hline Riqueza & $-0,033^{\star \star \star}$ & $-0,029^{\star \star \star}$ & $0,023^{\star \star \star}$ & $0,038^{\star \star \star}$ \\
\hline Propiedad de tierras & $0,374^{\star \star *}$ & $-0,162^{\star \star \star}$ & $-0,024^{\star \star \star}$ & $-0,142^{\star \star \star}$ \\
\hline \multicolumn{5}{|l|}{ Variables parroquiales y regionales } \\
\hline Electricidad & $-0,488^{* \star *}$ & $-0,374$ & 0,033 & $0,492^{\star \star \star}$ \\
\hline Teléfono & $-0,416^{\star \star \star}$ & $-0,065^{\star \star \star}$ & $0,100^{\star \star \star}$ & $0,382^{\star \star \star}$ \\
\hline Distancia 1 & $0,010^{\text {***}}$ & $-0,015^{\star \star \star}$ & 0,003 & 0,001 \\
\hline Distancia 2 & $0,010^{\star \star \star}$ & $-0,010^{\star \star \star}$ & 0,000 & 0,000 \\
\hline Distancia 3 & $0,011^{* \star \star}$ & 0,004 & $-0,005^{\star \star \star}$ & $-0,010^{\star * \star}$ \\
\hline Distancia 4 & $-0,004$ & 0,000 & $-0,000$ & 0,004 \\
\hline Costa & $-0,291$ & $0,059^{\star * \star}$ & $0,065^{\star \star \star}$ & $0,166^{\star \star \star}$ \\
\hline Oriente & $-0,119$ & $-0,023$ & $0,033^{* *}$ & $0,110^{\star \star \star}$ \\
\hline Número de observaciones & 16014 & & & \\
\hline Prueba de Wald $\chi^{2}$ & 9076 & & & \\
\hline Logaritmo de la función de verosimilitud & -15670 & & & \\
\hline
\end{tabular}

Fuente: Elaboración propia, sobre la base de datos de la Encuesta Nacional de Empleo, Desempleo y Subempleo, diciembre de 2010 y Censo de Población y Vivienda 2010.

Nota: $\quad{ }^{* \star *}$ significativo al $1 \%$; ${ }^{* *}$ significativo al $5 \%$; ${ }^{*}$ significativo al $10 \%$.

Como era de esperar, la educación presenta una correlación negativa con la probabilidad de que el empleo asalariado agrícola constituya la ocupación principal. Las personas que completaron la educación primaria, secundaria o superior son menos propensas a desempeñarse como trabajadores asalariados agrícolas. Los efectos de la educación en el autoempleo no agrícola son los esperados. Mientras que la probabilidad de participar en esa categoría de trabajo es mayor para las personas que completaron la educación primaria que para aquellos sin instrucción, esta se reduce drásticamente en el caso de quienes tienen un título universitario. Esto refleja que las dotaciones de educación requeridas para ese tipo de actividades son relativamente bajas. La probabilidad de participar en el 
empleo asalariado no agrícola aumenta en el caso de las personas que completaron la educación secundaria o superior, lo cual refleja que en el Ecuador rural, los retornos a la educación se encuentran solamente en esa categoría.

Los resultados también muestran que los jefes de hogar son menos propensos a desempeñarse como trabajadores asalariados agrícolas. Por otra parte, ser indígena aumenta la probabilidad de que el autoempleo agrícola sea la principal fuente de ingresos y reduce la de ser un trabajador asalariado. Esto concuerda con investigaciones previas (Vasco, 2013a y 2014), en las que se concluyó que los pueblos indígenas dependen principalmente del trabajo recíproco para satisfacer sus necesidades de mano de obra. Pertenecer al grupo étnico montubio aumenta la probabilidad de participar en el autoempleo agrícola y el empleo asalariado agrícola, por una parte, mientras que reduce la probabilidad de participar en cualquier tipo de empleo no agrícola, por otra. Estos resultados no sorprenden visto que este grupo se ha dedicado tradicionalmente a la agricultura.

Las personas de los hogares con más hombres adultos son más propensas a desempeñarse como trabajadores asalariados agrícolas. Esto puede obedecer a que los miembros de los hogares con flexibilidad laboral diversifican sus ingresos dedicándose al empleo asalariado agrícola. Por el contrario, las personas de los hogares con más hombres adultos son menos propensas a tomar parte en el autoempleo no agrícola y el empleo asalariado no agrícola. El número de mujeres adultas en un hogar presenta una correlación negativa con la probabilidad de participación en el autoempleo agrícola y el autoempleo no agrícola y una correlación positiva con la probabilidad de ser un trabajador asalariado no agrícola. Que haya más mujeres dedicadas al autoempleo agrícola o al autoempleo no agrícola permite que otros miembros del hogar se dediquen al empleo asalariado no agrícola.

Las personas de los hogares más ricos son menos propensas a participar en el trabajo agrícola y más propensas a realizar actividades no agrícolas. Los hogares más ricos están en mejores condiciones que sus pares más pobres para superar las barreras al ingreso que la participación en el ERNA a menudo supone (derechos de licencias, adquisición de equipos, entre otras) (Reardon y otros, 2000). La disponibilidad de tierras presenta una correlación positiva con la probabilidad de dedicarse al trabajo (agrícola y no agrícola) fuera del predio. En general, estos resultados indican que la falta de tierras es un factor que impulsa a la población rural a las actividades fuera del predio, ya sean agrícolas o de otro tipo. Las personas más instruidas pueden acceder a empleos no agrícolas mejor remunerados, mientras que el empleo asalariado agrícola es la opción disponible para la población que carece de tierras y de instrucción.

La disponibilidad de electricidad o teléfono presenta una correlación negativa con la probabilidad de participar en el autoempleo agrícola y el empleo asalariado agrícola, un hecho que no sorprende visto que estos servicios están disponibles sobre todo en las zonas urbanas. Por el contrario, las personas que residen en parroquias donde la electricidad y la telefonía están disponibles son más propensas a dedicarse al autoempleo no agrícola y al empleo asalariado no agrícola. Esto concuerda con investigaciones previas, en las que se concluye que la infraestructura desempeña un papel fundamental en el incremento de las oportunidades de empleo no agrícolas en las áreas rurales (Elbers y Lanjouw, 2001; Vasco, 2013b).

Como era de esperar, el autoempleo agrícola es más común en los centros poblados remotos, con excepción de las ciudades de más de 500.000 habitantes. En el caso del empleo asalariado agrícola, solo la distancia 1 (distancia hasta la ciudad más cercana con 50.000-100.000 habitantes) y la distancia 2 (distancia hasta la ciudad más cercana con 100.000-250.000 habitantes) fueron significativas y presentaron el signo negativo esperado. Estos resultados indican que la mayoría de las explotaciones agrícolas que están en condiciones de contratar mano de obra, y en consecuencia los mercados de trabajo agrícola más dinámicos, se concentran cerca de las ciudades pequeñas y no de las ciudades grandes. En el caso del empleo no agrícola (tanto por cuenta propia como asalariado), la distancia 3 (distancia hasta las ciudades de 250.000-500.000 habitantes) es significativa 
y presenta el signo negativo esperado. Una posible explicación para este resultado es que el grupo de ciudades a distancia 3 incluye tres ciudades medianas, entre ellas Santo Domingo de los Tsachilas (368.000 habitantes) y Ambato (330.000 habitantes). Aunque con diferentes patrones, estas ciudades presentan tres características comunes: tienen poblaciones relativamente grandes, posiciones geográficas ventajosas y vínculos estrechos con el sector agrícola. Ambato es la capital de la provincia de Tungurahua y se considera un modelo de ERNA exitoso en el contexto desfavorecido de la Sierra central (North y Cameron, 2000). La ventajosa posición de Tungurahua en el centro del país ha sido favorable para el comercio de productos agrícolas con Quito y Guayaquil, las dos ciudades más grandes del país (Ospina, 2010). Además, en el interior de Ambato hay una gran concentración de empresas familiares y pequeñas fábricas de cuero, productos textiles y de madera que dan empleo a gran parte de la población rural de Tungurahua. Santo Domingo de los Tsachilas es una ciudad subtropical que conecta Quito, la capital del país, con las principales ciudades de la Costa, entre ellas Guayaquil. La ganadería y la producción de aceite de palma, bananas y cacao, entre otros cultivos tropicales, han impulsado a las economías locales y regionales. Estos resultados concuerdan con el argumento de Reardon y otros (2000) de que el empleo no agrícola crece junto con un sector agrícola dinámico y con la afirmación de Jonasson y Helfand (2010) de que el tamaño de los mercados y la distancia con respecto a ellos son importantes para la participación en las actividades no agrícolas.

Los habitantes de la Costa tienen más probabilidades de trabajar como asalariados agrícolas que sus pares en las otras regiones. Esto concuerda con el hallazgo relativo a los montubios. Como se mencionó anteriormente, la producción en gran escala de cultivos comerciales tropicales (bananas, palma aceitera, caña de azúcar y cacao) absorbe un gran porcentaje de la población rural de esa región. Las personas que viven en la Costa y el Oriente son más propensas a obtener sus ingresos de fuentes no agrícolas que sus pares establecidos en la Sierra. En el caso de la Costa, la diferencia puede relacionarse con los patrones de propiedad de la tierra. Mientras que el 53\% de los hogares en la Sierra son propietarios de tierra, este porcentaje se reduce al 31\% en la Costa. La concentración de la tierra parece desempeñar un papel determinante en el impulso de los habitantes rurales de la Costa hacia el empleo asalariado agrícola y el ERNA en general.

El caso del Oriente es distinto, pues los hogares sin tierras representan el $45 \%$ de la muestra de esta región. ¿Por qué es más probable que las personas se dediquen al ERNA en una región que ha sido colonizada hace relativamente poco $^{4}$ y donde la concentración de la tierra no parece ser tan pronunciada como en la Costa? La respuesta a esta pregunta puede estar relacionada con la calidad de la tierra. La Amazonia ecuatoriana, localmente conocida como el Oriente, es una importante área de biodiversidad a nivel mundial (Myers y otros, 2000). Sin embargo, los suelos son muy frágiles y se agotan rápidamente después de que se retira la vegetación (Hicks y otros, 1990). En esas condiciones, como se muestra en el cuadro 3, los rendimientos de la agricultura son bajos y se necesitan fuentes de ingresos alternativas. Esto concuerda con investigaciones previas en las que se informaba de un considerable aumento de la proporción de personas dedicadas al trabajo fuera del predio agrícola en las provincias del Oriente (Bilsborrow, Barbieri y Pan, 2004; Vasco Pérez, Bilsborrow y Torres, 2015). De acuerdo con esos autores, esto obedece tanto a la reducción del tamaño de las explotaciones agrícolas debido a la continua subdivisión como al aumento de las oportunidades de empleo en las áreas urbanas.

En el cuadro 5 se muestran los resultados de la segunda etapa del método de corrección de Dubin y McFadden. Conforme varios estudios (Isgut, 2004; Jonasson y Helfand, 2010; Atamanova y Van den Berg, 2012), las variables de identificación utilizadas para consturir los términos de corrección de la selección son el sexo del jefe del hogar y las variables de composición del hogar. En estos estudios se asume que estas variables no influyen en los ingresos de cada categoría de empleo.

4 Las corrientes de colonización desde la Costa y especialmente desde la Sierra hacia el Oriente comenzaron en la década de 1970, después del descubrimiento de yacimientos petroleros en 1967. 


\section{Cuadro 5}

Ecuador: ingresos por categoría de empleo en las áreas rurales (En logaritmos)

\begin{tabular}{|c|c|c|c|c|}
\hline Variable & $\begin{array}{l}\text { Autoempleo } \\
\text { agrícola }\end{array}$ & $\begin{array}{l}\text { Empleo asalariado } \\
\text { agrícola }\end{array}$ & $\begin{array}{c}\text { Autoempleo } \\
\text { no agrícola }\end{array}$ & $\begin{array}{c}\text { Empleo asalariado } \\
\text { no agrícola }\end{array}$ \\
\hline \multicolumn{5}{|l|}{ Variables individuales } \\
\hline Edad & $0,018^{\star \star \star}$ & $0,014^{\star \star \star}$ & $0,038^{\star \star \star}$ & $0,028^{\star \star \star}$ \\
\hline Edad al cuadrado & $-0,000^{\star \star \star}$ & $-0,000^{\star \star \star}$ & $-0,000^{\star \star \star}$ & $-0,000^{\star \star \star}$ \\
\hline Mujer & $-0,340^{\star \star \star}$ & $-0,225^{\star \star *}$ & $-0,659^{\star \star *}$ & $-0,303^{\star * *}$ \\
\hline Jefatura del hogar & $0,233^{\star \star \star}$ & $0,170^{\star \star \star}$ & $0,165^{\star \star}$ & $0,203^{\star \star \star}$ \\
\hline Educación primaria & $0,112^{\star \star \star}$ & $-0,150$ & $-0,030$ & $-0,031$ \\
\hline Educación secundaria & $0,450^{\star \star \star}$ & 0,049 & 0,226 & $0,161^{\star \star \star}$ \\
\hline Educación universitaria & 0,335 & $-0,380$ & 0,310 & $0,470^{\star \star \star}$ \\
\hline Indígena & 0,000 & $-0,105$ & $-0,191$ & $-0,044$ \\
\hline Afroecuatoriano & $-0,231^{* \star *}$ & 0,017 & $-0,161$ & $-0,117$ \\
\hline Montubio & 0,007 & $-0,107^{\star \star \star}$ & $-0,023$ & 0,039 \\
\hline Blanco & 0,083 & 0,065 & $-0,074$ & $-0,056$ \\
\hline \multicolumn{5}{|l|}{ Variables del hogar } \\
\hline Riqueza & $0,104^{\star \star \star}$ & $0,065^{\star \star \star}$ & $0,133^{\star \star \star}$ & $0,059^{\star \star \star}$ \\
\hline Propiedad de tierras & $0,240^{*}$ & $-0,010$ & 0,128 & $0,156^{\star \star \star}$ \\
\hline \multicolumn{5}{|c|}{ Variables parroquiales y regionales } \\
\hline Electricidad & $-0,860^{\star \star \star}$ & $0,417^{\star \star \star}$ & $0,576^{\star}$ & 0,034 \\
\hline Teléfono & $-0,365^{\star}$ & $-0,051$ & $-0,398$ & $-0,021$ \\
\hline Distancia 1 & $-0,035^{\star \star \star}$ & $-0,009$ & $-0,002$ & 0,002 \\
\hline Distancia 2 & 0,005 & $-0,018^{\star \star \star}$ & 0,009 & $0,012^{\star \star}$ \\
\hline Distancia 3 & 0,008 & $-0,022^{\star \star \star}$ & $-0,017$ & $0,014^{\star *}$ \\
\hline Distancia 4 & $-0,111^{\star \star \star}$ & $-0,014$ & 0,021 & $-0,011$ \\
\hline Costa & 0,162 & $0,310^{\star \star \star}$ & 0,136 & 0,022 \\
\hline Oriente & $-0,079$ & $0,201^{\star \star \star}$ & $0,251^{*}$ & $0,217^{\star \star \star}$ \\
\hline$m_{1}$ & 0,119 & $-0,109$ & $-0,045$ & 0,185 \\
\hline $\mathrm{m}_{2}$ & $-0,535$ & $-0,053$ & $-0,086$ & $-0,126$ \\
\hline$m_{3}$ & $-0,918$ & $-0,090$ & $-0,214^{\star \star}$ & 0,097 \\
\hline$m_{4}$ & 0,321 & 0,099 & $-0,289$ & $-0,216$ \\
\hline Estadísticas F & $57,50^{\star \star \star}$ & $25,13^{\star \star \star}$ & $26,07^{\star \star \star}$ & $56,11^{\star \star \star}$ \\
\hline $\mathrm{R}^{2}$ & 0,252 & 0,158 & 0,292 & 0,292 \\
\hline
\end{tabular}

Fuente: Elaboración propia, sobre la base de datos de la Encuesta Nacional de Empleo, Desempleo y Subempleo, diciembre de 2010 y Censo de Población y Vivienda 2010.

Nota: $\mathrm{m} 1, \mathrm{~m} 2, \mathrm{~m} 3$ y $\mathrm{m} 4$ son los términos de corrección de selección estimados a partir del modelo de selección. ${ }^{\star * *}$ significativo al $1 \%$; ${ }^{* *}$ significativo al $5 \%$; ${ }^{*}$ significativo al $10 \%$.

Ser mujer reduce los ingresos derivados del autoempleo agrícola, el empleo asalariado agrícola, el autoempleo no agrícola y el empleo asalariado no agrícola en un $34 \%$, un $20 \%$, un $48 \%$ y un $26 \%$, respectivamente. En el caso del trabajo no agrícola, este resultado es coherente con investigaciones previas (Elbers y Lanjouw, 2001), en las que se informaba que las mujeres se concentraban en empleos no agrícolas de bajos ingresos. Los rendimientos de la educación solo se encuentran con respecto al empleo asalariado agrícola (educación primaria y secundaria) y al empleo asalariado no agrícola (educación secundaria y universtaria). Haber completado la educación secundaria o superior aumenta los ingresos de esta última categoría un $17 \%$ y un $60 \%$, respectivamente. Por el contrario, ninguna variable ficticia de educación tuvo un efecto significativo en los ingresos derivados del autoempleo 
no agrícola ${ }^{5}$. Este resultado apoya el argumento de que, si no intervienen otros factores, ese tipo de empleo no requiere altas dotaciones en materia de educación. En general, los jefes de hogar ganan más que otros miembros del hogar en todas las categorías de empleo. En cuanto a las variables relativas a la etnia, las personas autodefinidas como afroecuatorianas cuya principal ocupación corresponde al autoempleo agrícola ganan un $23 \%$ menos que sus pares mestizos, mientras que los montubios ganan un $11 \%$ menos que sus pares mestizos en el empleo asalariado agrícola, incluso aunque son más propensos a dedicarse a ese tipo de trabajo. No hay diferencias significativas entre los grupos étnicos en lo que respecta a los ingresos no agrícolas.

La riqueza presenta una correlación positiva con ingresos más altos en todas las categorías de empleo. Los hogares más ricos están en mejores condiciones para acceder a la tecnología agrícola (fertilizantes químicos, irrigación y mecanización), que contribuye a incrementar la producción y en consecuencia los ingresos. En forma análoga, las personas de los hogares más ricos tienen los medios para superar las barreras al ingreso que supone el autoempleo no agrícola, como la necesidad de capital inicial y el costo de derechos de licencias y maquinaria. En el caso del empleo asalariado, este resultado puede reflejar el hecho de que los miembros de los hogares más ricos tienen más capital social y en consecuencia pueden acceder a empleos no agrícolas mejor remunerados (Jonasson y Helfand, 2010). Un efecto similar puede existir con respecto a la propiedad de tierras, que se asocia con ingresos más altos para los trabajadores no agrícolas. Como señalan Lanjouw y Stern (1998), el acceso a empleos no agrícolas atractivos puede estar determinado por la riqueza, que a su vez puede estar vinculada con la propiedad de tierras.

Las mayores proporciones de acceso a electricidad se asocian con menores ingresos en la categoría del autoempleo agrícola. Esto puede obedecer a que las explotaciones agrícolas familiares más grandes y los agricultores que dependen completamente de ese tipo de trabajo normalmente se encuentran en lugares donde la disponibilidad del servicio es baja. En contraste, los ingresos más altos del empleo asalariado agrícola se asocian con mayores proporciones de acceso a la electricidad. Esto puede deberse a que la producción de bienes intensivos en mano de obra (como la producción de flores) es también intensiva en el uso de electricidad. Los ingresos derivados del autoempleo no agrícola son mayores en las parroquias con grandes proporciones de acceso a la electricidad: un incremento del $10 \%$ en este valor supone un aumento de los ingresos del autoempleo del 6\%. El carácter no significativo en el caso del empleo asalariado no agrícola puede indicar que el acceso a la electricidad es una condición esencial para el funcionamiento de las empresas suficientemente grandes para contratar mano de obra, de manera que no desempeña ningún papel en la determinación de los salarios.

El autoempleo agrícola es mayor cerca de las ciudades a distancia 1 y distancia 4. En el caso de la distancia 1, esto puede obedecer a que las ciudades pequeñas constituyen el principal mercado para los pequeños agricultores que venden sus productos a los comerciantes. En el caso de la distancia 4, los ingresos son mayores para aquellos que pueden vender sus productos en las grandes ciudades incluidas en esta categoría. El empleo asalariado agrícola se paga mejor en las áreas cerca de las ciudades a distancia 2 y a distancia 3. Una posible explicación para estos resultados es que las empresas agrícolas más desarrolladas, que ofrecen mejores salarios en comparación con las de pequeña escala, se encuentran en esas áreas. Cuanto más lejos estén las ciudades de distancia 2 y distancia 3, mayores serán los ingresos del empleo asalariado no agrícola. Esto puede deberse a que la competencia por empleos en las áreas urbanas más grandes puede ser mayor, manteniendo los salarios más bajos que en las zonas más alejadas de las grandes ciudades.

\footnotetext{
5 Los efectos de la educación continúan siendo no significativos, incluso cuando las variables ficticias de educación se reemplazan por el número de años de educación formal y su término al cuadrado.
} 
Los salarios de los trabajadores agrícolas son más altos en la Costa y en el Oriente. Estos resultados coinciden con las cifras presentadas en el cuadro 3. Si no intervienen otros factores, los ingresos del empleo no agrícola son mayores en el Oriente que en la Sierra. Como se mencionó anteriormente, las compañías petroleras, que pagan los salarios no agrícolas más altos, están situadas en el Oriente.

\section{Conclusiones}

El empleo no agrícola es una importante fuente de ingresos para la población rural del Ecuador, pues representa el 36\% de los ingresos de los hogares y emplea alrededor del 33\% de la mano de obra rural del país. En este estudio se examinaron los determinantes de la participación en el empleo no agrícola y de los ingresos no agrícolas. El análisis empírico muestra que las mujeres son más propensas que los hombres a dedicarse al autoempleo no agrícola. Sin embargo, ganan menos que los hombres, tanto en ese tipo de empleo como en el empleo asalariado no agrícola. La participación en esta última categoría es más común entre las personas instruidas, que generalmente también cuentan con el capital físico necesario para superar las barreras de ingreso que el ERNA supone. Se observa una marcada tendencia a que las personas que carecen de tierras se dediquen al ERNA. Como era de esperar, los resultados también muestran que la participación en el autoempleo no agrícola no requiere altas dotaciones educativas. Esto evidencia que todavía se trata de un sector incipiente.

Se puso énfasis en las variables geográficas y de localización. Los resultados sugieren que el ERNA florece en las áreas cerca de las ciudades medianas que cuentan con un sector agrícola dinámico. La probabilidad de participar en el trabajo no agrícola es mayor en la Costa y en el Oriente que en la Sierra. Entre las distintas razones para ello, se destacan la concentración de la tierra en el caso de la Costa y la calidad de la tierra en el caso del Oriente.

Se observan variaciones regionales en el diferencial de salarios entre los ingresos agrícolas y no agrícolas. Mientras que los ingresos agrícolas son mucho menores que los del empleo no agrícola en la Sierra y el Oriente, quienes se dedican al autoempleo agrícola como principal ocupación en la Costa ganan más que la mayoría de los trabajadores en la categoría del autoempleo no agrícola. Sin embargo, el empleo asalariado no agrícola ofrece mayores ingresos que cualquier forma de trabajo agrícola, lo que indica su potencial para mitigar la pobreza en las zonas rurales. No obstante, la participación en el ERNA en general y en el ERNA bien remunerado en particular requiere altas dotaciones de capital humano y físico que rara vez están disponibles para los pobres. Las políticas para abordar esta situación deberían concentrarse en brindar educación, capacitación y crédito a la población rural para superar las barreras que impiden a los pobres el acceso al empleo no agrícola.

Debido a que la participación en el empleo no agrícola no solo está determinada por la distancia con respecto a los mercados sino también por el tamaño de los mercados y el ambiente económico local, las políticas también deberían promover el desarrollo de las ciudades medianas, que -como se mostró anteriormente - tienen el potencial para absorber el excedente de mano de obra rural. Las inversiones en infraestructura rural, la promoción de la agricultura y el turismo y los incentivos fiscales para las empresas dispuestas a trasladarse a áreas rurales desfavorecidas podrían ser útiles para lograr ese objetivo.

\section{Bibliografía}

Atamanova, A. y M. Van den Berg (2012), "Participation and returns in rural nonfarm activities: evidence from the Kyrgyz Republic", Agricultural Economics, vol. 43, № 4, International Association of Agricultural Economists. 
Berdegué, J. y otros (2001), "Rural nonfarm employment and incomes in Chile", World Development, vol. 29, $N^{\circ} 3$, Amsterdam, Elsevier.

Bilsborrow, R., A. Barbieri y W. Pan (2004), "Changes in population and land use over time in the Ecuadorian Amazon", Acta Amazonica, vol. 34, № 4.

Bourguignon, F., M. Fournier y M. Gurgand (2007), "Selection bias corrections based on the multinomial logit model: Monte Carlo comparisons", Journal of Economic Surveys, vol. 21, № 1, Wiley.

Dubin, J.A. y D.L. McFadden (1984), "An econometric analysis of residential electric appliance holdings and consumption", Econometrica, vol. 52, № 2, Nueva York, The Econometric Society.

Elbers, C. y P. Lanjouw (2001), "Intersectoral transfer, growth and inequality in rural Ecuador", World Development, vol. 29, №3, Amsterdam, Elsevier.

Ellis, F. (2000), "The determinants of rural livelihood diversification in developing countries", Journal of Agricultural Economics, vol. 51, № 2, Agricultural Economics Society.

(1999), "Rural livelihood diversity in developing countries: evidence and policy implications", Natural Resource Perspectives, № 40, Overseas Development Institute.

(1993), Peasant Economics: Farm Households and Agrarian Development, Cambridge, Cambridge University Press.

Ferreira, F. y P. Lanjouw (2001), "Rural nonfarm activities and poverty in the Brazilian Northeast", World Development, vol. 29, №3, Amsterdam, Elsevier.

Haggblade, S., P. Hazell y T. Reardon (2010), "The rural non-farm economy: prospects for growth and poverty reduction", World Development, vol. 38, № 10, Amsterdam, Elsevier.

Hicks, J.F. y otros (1990), Ecuador's Amazon Region Development Issues and Options, Washington, D.C., Banco Mundial.

Isgut, A. (2004), "Non-farm income and employment in rural Honduras: assessing the role of locational factors", Journal of Development Studies, vol. 40, №3, Taylor \& Francis.

Jonasson, E. y S.M. Helfand (2010), "How important are locational characteristics for rural non-agricultural employment? Lessons from Brazil", World Development, vol. 38, № 5, Amsterdam, Elsevier.

Köbrich, C. y M. Dirven (2007), "Características del empleo rural no agrícola en América Latina con énfasis en los servicios", serie Desarrollo Productivo, № 174 (LC/L.2659-P), Santiago, Comisión Económica para América Latina y el Caribe (CEPAL).

Lanjouw, P. (2001), "Nonfarm employment and poverty in rural El Salvador", World Development, vol. 29, $N^{\circ} 3$, Amsterdam, Elsevier.

(1999), "Rural nonagricultural employment and poverty in Ecuador", Economic Development and Cultural Change, vol. 48, $N^{\circ} 1$, Chicago, The University of Chicago Press.

Lanjouw, J.O. y P. Lanjouw (2001), "The rural non-farm sector: issues and evidence from developing countries", Agricultural Economics, vol. 26, $\mathrm{N}^{\circ}$ 1, International Association of Agricultural Economists.

Lanjouw, P. y N. Stern (1998), A Kind of Growth: Palanpur 1957-1993, Oxford, Oxford University Press.

Laszlo, S. (2005), "Self-employment earnings and returns to education in rural Peru", Journal of Development Studies, vol. 41, № 7, Taylor \& Francis.

Martínez, L. (2004), "El campesino andino y la globalización a fines de siglo (una mirada sobre el caso ecuatoriano)", Revista Europea de Estudios Latinoamericanos y del Caribe, Nㅜ 77.

(2002), Economía política de las comunidades indígenas, Quito, Instituto Latinoamericano de Investigaciones Sociales (ILDIS).

(2000), "La especificidad del empleo rural", Antología de estudios rurales, A. Torres (eds.), Quito, FLACSO Ecuador/Instituto Latinoamericano de Investigaciones Sociales (ILDIS).

McFadden, D. (1973), "Conditional logit analysis of qualitative choice behavior", Frontiers in Econometrics, P. Zarembka (ed.), Nueva York, Academic Press.

Myers, N. y otros (2000), "Biodiversity hotspots for conservation priorities", Nature, № 403.

North, L.L. y J.D. Cameron (2000), "Grassroots-based rural development strategies: Ecuador in comparative perspective", World Development, vol. 28, № 10, Amsterdam, Elsevier.

Ospina, P. (2010), "Tungurahua rural: el territorio de senderos que se bifurcan”, Ecuador Debate, vol. 81, $N^{\circ}$ 3, Quito, Centro Andino de Acción Popular.

Reardon, T. y otros (2000), "Household income diversification into rural nonfarm activities", Transforming the Rural Nonfarm Economy, S. Haggblade, P. Hazell y T. Reardon (eds.), Baltimore, Johns Hopkins University Press.

Vasco, C. (2014), "Reciprocal and wage labour in rural Ecuador. A quantitative analysis", Journal of Agriculture and Rural Development in the Tropics and Subtropics, vol. 115, № 1. 
(2013a), "Factores determinantes del trabajo recíproco y del uso de mano de obra salariada en el Ecuador rural", Eutopía, № 4, Quito, FLACSO-Ecuador.

- (2013b), "Migration, remittances and entrepreneurship: the case of rural Ecuador", Migraciones Internacionales, vol. 7, № 1, Ciudad de México, El Colegio de la Frontera Norte.

Vasco Pérez, C., R. Bilsborrow y B. Torres (2015), "Income diversification of migrant colonists vs. indigenous populations: contrasting strategies in the Amazon", Journal of Rural Studies, vol. 42, Amsterdam, Elsevier. 